\title{
String Theory and Loop Space Index Theorems *
}

\author{
Orlando Alvarez ${ }^{1}$, T.-P. Killingback ${ }^{2}$, Michelangelo Mangano ${ }^{3}$, \\ and Paul Windey ${ }^{1}$ \\ 1 Department of Physics and Lawrence Berkeley Laboratory, University of California, Berkeley, \\ CA 94720 , USA \\ 2 DAMTP, University of Cambridge, England CB3 9EW, United Kingdom \\ ${ }^{3}$ Fermi National Accelerator Laboratory, Batavia, IL 60510, USA
}

\begin{abstract}
We study index theorems for the Dirac-Ramond operator on a compact Riemannian manifold. The existence of a group action on the loop space makes possible the definition of a character valued index which we calculate by using a two-dimensional sigma model with $N=1 / 2$ supersymmetry. We compute the Euler characteristic, the Hirzebruch signature and the Dirac-Ramond genus of loop space. We compare our results to the calculations made by using the Atiyah-Singer character-valued index theorem.
\end{abstract}

In this paper we will discuss some attempts at calculating the index of some differential operators acting on infinite dimensional spaces. In particular we will study some of the topological properties of the twisted Dirac-Ramond operator on the loop space $\mathscr{L}(M)$ of a compact Riemannian manifold $M$. The first problem that one has to face in studying the index on an infinite dimensional manifold is that the kernel of the operator may be infinite dimensional. One therefore needs a device which will split this infinite degeneracy and allow one to work with finite dimensional subspaces of the kernel. A natural way of dealing with this obstacle is to look for a character valued index ( $G$-index) $[1-4]$ which will split the kernel into finite dimensional pieces transforming according to different representations of some group. Witten [5] has emphasized that the equivariant Dirac-Ramond operator provides such an example. The loop space $\mathscr{L}(M)$ has an $S^{1}$ action given by sending the loop parameter $\sigma$ to $\sigma+\Delta$, where $\Delta$ is a constant. Representations of $S^{1}$ are labelled by an integer $n$ which corresponds to the two-dimensional momentum of the string state. In the Ramond case, the number of states for each value of $n$ is finite.

\footnotetext{
* This work was supported in part by the Director, Office of Energy Research, Office of High Energy and Nuclear Physics, Division of High Energy Physics of the U.S. Department of Energy under Contract DE-AC03-76SF00098 and in part by the National Science Foundation under grant PHY85-15857. Fermilab is operated by the Universities Research Association, Inc., under contract with the United States Department of Energy
} 
The index is studied by generalizing the supersymmetric quantum mechanics methods $[6,7]$ that have been useful in understanding the Atiyah-Singer index theorem ${ }^{1}[4]$. In analogy to the Dirac operator coupled to a vector bundle $V$ we can isolate the fundamental object as the generalization of the pure Dirac operator. This is the Ramond operator which is the supersymmetry generator for a chiral right moving $N=1 / 2$ string theory. The vector bundle enters through the left moving sector of the theory. By suitable choice of the left moving sector we can compute indices of operators which lead to infinite dimensional generalization of the Euler characteristic and the Hirzebruch signature.

A feature of the character valued indices on finite dimensional manifolds is that they may be computed by looking at the fixed point set of the group action. In the case of the loop space $\mathscr{L}(M)$ the fixed point set of the $S^{1}$ action is just the set of constant loops which is the manifold $M$ itself. If the character valued formulas of Atiyah and Singer are valid in infinite dimensions then one would expect that the index formulas should involve integrals over the manifold $M$ and not integrals over the infinite dimensional loop space $\mathscr{L}(M)$. We find this to be true in the cases we have studied. By formal manipulations of the character valued index theorem of Atiyah and Singer we have found agreement with our formulas.

The study of the Dirac-Ramond operator $[5,9]$ has been discussed in detail by Witten [10] who related the spectrum of string theory to elliptic genera. In a nice series of papers Schellekens and Warner [11-13] deduced properties of the partition function in background field by the use of modularity properties. The Schellekens-Warner results were shown to be related to elliptic genera and the Ramond operator by Witten. In a follow-up paper Pilch, Schellekens, and Warner [14] discuss the relation of their results to the $N=1 / 2$ supersymmetric system. The mathematics of elliptic genera and related topics are discussed in [15-17].

The lagrangian for the $N=1 / 2$ two dimensional field theory in a background gravitational field is

$$
\mathscr{L}=g_{\mu \nu}(X) \bar{\partial} X^{\mu} \partial X^{\nu}+g_{\mu v}(X)\left[\bar{\partial} \psi^{\mu}+\bar{\partial} X^{\kappa} \Gamma_{\kappa \lambda}^{\mu}(X) \psi^{\lambda}\right] \psi^{\nu} .
$$

The supersymmetry charge for the above is the equivariant Ramond operator, i.e. a Dirac like operator plus the generator $g_{\mu \nu} d X^{v} / d \sigma$ of the $S^{1}$ translations [5]:

$$
Q=\int d \sigma \psi^{\mu}(\sigma)\left(-i \frac{D}{D X^{\mu}(\sigma)}+g_{\mu v}(X) \frac{d X^{v}}{d \sigma}\right) .
$$

The conditions under which this operator makes sense globally on $\mathscr{L}(M)$ have been studied in [18], where the concept of string structure is introduced ${ }^{2}$. There it is found that $Q$ can be consistently defined only if the first Pontrjagin class of $M$, $p_{1}(M)$, vanishes in integral cohomology. We will see later on that a similar obstruction arises in trying to define the index of $Q$ [9]. The index has a path integral representation [20]. We will compute the path integral for the above lagrangian with periodic boundary conditions on a skew torus with sides specified by 1 and $\tau=\tau_{1}+i \tau_{2}$ and $\tau_{2}>0$. According to Cardy [21] the path integral

\footnotetext{
${ }^{1}$ SUSY quantum mechanics proofs of the $G$-index theorem may be found in $[6,8]$

${ }^{2}$ The role of $p_{1}(M)=0$ is discussed by Freed [19] in a different context
} 
computes

$$
I=\operatorname{Tr}(-1)^{F} \exp \left(-2 \pi \tau_{2} H+2 \pi i \tau_{1} P\right),
$$

where $H$ is the Hamiltonian and $P$ is the momentum, i.e. the generator of the $S^{1}$ translations. Calculating this object gives an explicit representation for the character valued index. To see this we note that the Hamiltonian and the momentum may be written in the form

$$
\begin{aligned}
& H=\left(L_{0}+\varepsilon\right)+\left(\bar{L}_{0}+\bar{\varepsilon}\right), \\
& P=\left(L_{0}+\varepsilon\right)-\left(\bar{L}_{0}+\bar{\varepsilon}\right),
\end{aligned}
$$

where $\varepsilon=-c / 24$ and $\bar{\varepsilon}=-\bar{c} / 24, c$ and $\bar{c}$ being respectively the Virasoro central charges for the right and the left moving sectors. It is very important to notice that

$$
L_{0}+\varepsilon=Q^{2} \text {. }
$$

Equation (3) may be written in the following form

$$
I=\operatorname{Tr}(-1)^{F} q^{L_{0}+\varepsilon}(\bar{q})^{\bar{L}_{0}+\bar{\varepsilon}},
$$

where $q=\exp (2 \pi i \tau)$. Because $Q$ anticommutes with $(-1)^{F}$ one has a fermion number pairing of eigenstates of $Q^{2}$ whenever the $Q^{2}$ eigenvalue is greater than zero. The only terms that survive in the above trace are the states annihilated by $Q$, i.e. the supersymmetric states. It is easy to see that the above reduces to

$$
I=\operatorname{Tr}_{\operatorname{SUSY}}(-1)^{F}(\bar{q})^{-P} \text {. }
$$

The SUSY subscript denotes a restricted trace over the supersymmetric states. If the manifold $M$ were non-compact, as is the case when $M$ is the ordinary Euclidean or Minkowskian space-time, an infinite number of left moving states would contribute to the trace, because by a proper choice of the $d$-dimensional momentum, whose spectrum is continuous, we may always manage to obtain $L_{0}+\varepsilon=0$ at any level of the excited spectrum. However, when $M$ is compact the spectrum of the Beltrami-Laplace operator is discrete, and in general only the ground state $(p=0)$ can be supersymmetric. It is certainly possible that by accident the spectrum of momenta contains a proper eigenvalue, as to allow $L_{0}+\varepsilon=0$ for some state other than the vacuum. However, since we are calculating topological invariants these states cannot contribute, because a small change in the metric of $M$ would remove the accidental degeneracy, while leaving the trace invariant.

If the eigenvalues of $P$ are $\lambda$ then (8) reduces to

$$
I=\sum_{\lambda} I_{\lambda}(\bar{q})^{-\lambda}
$$

where $I_{\lambda}$ is the index on the subspace with momentum $\lambda$. Notice that the above is a character valued index where the entire kernel of $Q$ is split into the associated $P$ eigenspaces. Notice that states in the kernel of $Q$ may have any allowed $\bar{L}_{0}+\bar{\varepsilon}$ eigenvalue. In the examples taken from string theory the eigenvalues $\lambda$ are of the form $-(\bar{\varepsilon}+n)$, where $n$ is a non-negative integer. In conformal field theories the eigenvalues $\lambda$ will be of the form $-(\bar{\varepsilon}+\bar{h}+n)$, where $\bar{h}$ is the conformal weight of the highest weight vectors used to construct the various Verma modules in the 
spectrum of the theory. For example, one could have a complicated character sum of the form

$$
I=(\bar{q})^{\bar{\varepsilon}+\bar{h}_{1}} \sum_{n=0}^{\infty} a_{n}(\bar{q})^{n}+(\bar{q})^{\bar{\varepsilon}+\bar{h}_{2}} \sum_{n=0}^{\infty} b_{n}(\bar{q})^{n} .
$$

It is interesting to notice that contrarily to what happens for non-chiral supersymmetries, states with $H \neq 0$ contribute to the trace. Furthermore, for these states the relation $H=-P$ holds. We can express (8) as follows:

$$
I=\operatorname{Tr}(-1)^{F} e^{2 \pi i \tau_{1} P} e^{-2 \pi \tau_{2} H} .
$$

Since $H$ is the generator of a group action (the Hamiltonian flow for loops in loopspace), we may interpret this representation as the character valued index with respect to simultaneous $H$ and $P$ eigenvectors, even though the flow generated by $H$ is non-compact. This observation will be relevant when comparing the result of the path-integral calculation of the index with the abstract formulation of the character-valued index theorems.

The index should just involve information about the loop space. Notice that $\tau_{2}$ has nothing to do with loops while $\tau_{1}$ is associated with the $S^{1}$ action. The character valued index should only be a function of $\tau_{1}$ but the expression (9) involves all of $\tau$. The crux of the matter is that the index in an analytic function of $\bar{q}$ and the character valued index is its boundary value. It is therefore possible to determine the index by studying the path integral in the small $\tau_{2}$ limit and then continuing the result to the associated analytic function.

To calculate the path integral in the small $\tau_{2}$ limit we need the quadratic approximation to the lagrangian:

$$
\mathscr{L}_{2}=\bar{\partial} X^{\mu} \partial X^{\mu}+\bar{\partial} \psi^{\mu} \cdot \psi^{\mu}+\mathscr{R}_{\mu \nu} \bar{\partial} X^{\mu} X^{\nu}
$$

where $X$ and $\psi$ are now interpreted as fluctuations about the classical constant solutions $X_{0}^{\mu}$ and $\psi_{0}^{\mu}$ and $\mathscr{R}_{\mu \nu}=\frac{1}{2} R_{\mu \nu \varrho \sigma} \psi_{0}^{\varrho} \psi_{0}^{\sigma}$.

A straightforward calculation shows that the index is given by

$$
I=\frac{1}{(2 \pi)^{d / 2}} \int d^{d} X_{0} d^{d} \psi_{0}\left[\operatorname{det}^{\prime}(-\partial \bar{\partial}+\mathscr{R} \bar{\partial})\right]^{-1 / 2}\left[\operatorname{det}^{\prime}(\bar{\partial})\right]^{1 / 2},
$$

where the prime denotes the omission of the zero modes. The calculation of the product of the determinants is a bit cumbersome, and is carried out in full detail in [22]. The result is

$$
\begin{aligned}
I= & \frac{1}{(2 \pi)^{d / 2}} \int d^{d} X_{0} d^{d} \psi_{0} \exp \left[\sum_{j} \sum_{\Lambda}^{\prime}\left(\frac{2 \tau_{2} i x_{j}}{m+n \bar{\tau}}\right)+\frac{1}{2} \sum_{j} \sum_{\Lambda}^{\prime}\left(\frac{2 \tau_{2} i x_{j}}{m+n \bar{\tau}}\right)^{2}\right] \\
& \times \prod_{j} \frac{2 \tau_{2} i x_{j}}{\sigma\left(2 \tau_{2} i x_{j} ; \bar{\tau}\right)} \cdot \frac{1}{\left(2 \tau_{2}\right)^{d / 2}} \cdot \frac{1}{\left[\eta_{-}(\bar{q})\right]^{d}} .
\end{aligned}
$$

$\Lambda$ is the period lattice determined by a parallelogram with sides 1 and $\tau$. The $x_{j}$ are the entries in the formal block diagonalization of the "matrix" $\mathscr{R}_{\mu v} / 2 \pi . \eta_{-}(\bar{q})$ is the usual Dedekind's function, and for later use we define

$$
\eta_{ \pm}(\bar{q})=(\bar{q})^{1 / 24} \prod_{1}^{\infty}\left(1 \pm(\bar{q})^{n}\right)
$$


The Weierstrass $\sigma$-function [23] is defined by

$$
\sigma(z ; \tau)=z \prod_{\Lambda}^{\prime}\left(1-\frac{z}{m+n \tau}\right) \exp \left[\sum_{\Lambda}^{\prime} \frac{z}{m+n \tau}+\frac{1}{2} \sum_{\Lambda}^{\prime}\left(\frac{z}{m+n \tau}\right)^{2}\right] .
$$

The prime in sums and products denotes the omission of the zero-mode. The $\sigma$ function is the generalization of the sine function to the elliptic case.

Because of the integration of the fermionic zero modes $\psi_{0}^{\mu}$ all that contributes is the term which is of order $d$ in $\psi$. One can verify that the above is equivalent to the following generating function:

$$
\begin{aligned}
I= & \int_{M} \exp \left[\sum_{j} \sum_{\Lambda} \frac{i x_{j} / 2 \pi}{m+n \bar{\tau}}+\frac{1}{2} \sum_{j} \sum_{\Lambda}^{\prime}\left(\frac{i x_{j} / 2 \pi}{m+n \bar{\tau}}\right)^{2}\right] \\
& \times \prod_{j} \frac{i x_{j} / 2 \pi}{\sigma\left(i x_{j} / 2 \pi ; \bar{\tau}\right)} \cdot \frac{1}{\left[\eta_{-}(\bar{q})\right]^{d}} .
\end{aligned}
$$

In the above we now interpret $\mathscr{R}$ as a differential two-form. Since the first class is given by $p_{1}(M)=\sum_{j} x_{j}^{2}$ we see that unless $p_{1}(M)$ vanishes the above will be divergent. The vanishing of $p_{1}(M)$ is the statement that $\operatorname{tr} \mathscr{R}^{2}=d \omega_{3}$. One can interpret this as being the statement that the index of the Ramond operator can only be well defined on manifolds with vanishing first real Pontrjagin class. This is a weaker condition than the ones discussed in $[18,19]$.

We would like to emphasize that the necessity of the vanishing of $p_{1}(M)$ is a pure stringlike phenomenon which is absent in any finite truncation of the infinite spectrum of the string theory. The reason is that the sum is logarithmically divergent and therefore all energy scales contribute the same. The highly excited states (large $|m|$ and large $|n|$ ) are just as important as the low lying states! One can easily see that any finite truncation of (13) does not necessitate the vanishing of the $p_{1}(M)$.

We discard a term of the form

$$
\sum_{j} x_{j} \sum_{\Lambda}^{\prime} \frac{1}{m+n \bar{\tau}}
$$

because any reasonable regulator should respect the symmetries of the period lattice. Additionally, one encounters both $x_{j}$ and $-x_{j}$ in the block diagonalization of $\mathscr{R}$, therefore such terms should be absent assuming one has a good regulator. There is a suggestion from looking at several of these calculations that complex manifolds $M$ with both $\sum x_{j}=c_{1}\left(T^{(1,0)}(M)\right)=0$ and $p_{1}(M)=0$ should have extremely well behaved indices due to the absence of ambiguities among different regularizations of the path integral. Paraphrasing one can say that it may be very interesting to study the index on the loop space of complex manifolds with vanishing first and second Chern classes of the holomorphic tangent bundle. We remark that these are related to the class of manifolds that arise in the compactification [24] of the heterotic string $[25,26]$. In this case the vanishing of the Pontrjagin class is replaced by the Green-Schwarz condition [27] $\operatorname{tr} \mathscr{R}^{2}-\operatorname{tr} F^{2}=0$. 
A few words are required about renormalizations and anomalies. The typical one loop renormalization of a $\sigma$-model is of the form $\log \Lambda \cdot R_{\mu \nu} \partial X_{0}^{\mu} \wedge \bar{\partial} X_{0}^{v}$ where $\Lambda$ is the cutoff [28]. In our calculation the background $X_{0}$ is constant and therefore such a term will not appear. This is important because the index is topological. The index may be defined whether or not the theory is conformal. Many anomaly features are hard to observe since the torus we are studying has a flat metric. Note that $\mathscr{R}$ is a $(1,0)$-form, therefore the logarithmically divergent $\operatorname{tr} \mathscr{R}^{2}$ term is not Lorentz or Einstein invariant. This is not surprising since this model has Lorentz and gravitational anomalies.

We may now show the equivalence between (17) and the formal expression obtained by applying the character valued index theorem to $\mathscr{L}(M)$. The general expression for this index on a manifold $N$ is:

$$
I_{g}=\int_{N_{b}} \prod_{j} \frac{i x_{j} / 2}{\sin i x_{j} / 2} \prod_{r} \frac{1}{2 i \sin \left(\bar{\theta}_{r} / 2\right)},
$$

where $N_{b}$ is the fixed point set of the group action and $\bar{\theta}_{r}=\alpha \theta_{r}+x_{r}$. The $\theta_{r}$ are the "eigenvalues" of the group action on $T N, x_{r}$ are the "eigenvalues" of the curvature two form at the fixed points, and $\alpha$ is the parameter of the infinitesimal transformation. In our case $N_{b}=\mathscr{L}(M)_{b}=M$ and $T\left(\mathscr{L}(M)_{b}\right)$ is the $\infty$-dimensional vector space generated by the string Fourier modes, $X_{n}^{\mu}$. By definition $\theta_{r}$ is $\theta_{n}^{\mu}=2 \pi n$ if $\alpha=\tau_{1}-i \tau_{2}=\bar{\tau}$ corresponds to the simultaneous $H$ and $P$ actions. Applying the character valued theorem we find

$$
\begin{aligned}
\prod_{r} \frac{1}{2 i \sin \left(\bar{\theta}_{r} / 2\right)}=\prod_{j} \prod_{n=1}^{\infty}\left(\frac{\exp \left(-\pi i \bar{\tau} n+x_{j} / 2\right)}{1-\exp \left(-2 \pi i \bar{\tau} n+x_{j}\right)}\right) \cdot\left(\frac{\exp \left(-\pi i \bar{\tau} n-x_{j} / 2\right)}{1-\exp \left(-2 \pi i \bar{\tau} n-x_{j}\right)}\right) \\
=\prod_{j} \prod_{n=1}^{\infty} \frac{(\bar{q})^{n}}{\left(1-(\bar{q})^{n} e^{x_{j}}\right)\left(1-(\bar{q})^{n} e^{-x_{j}}\right)} .
\end{aligned}
$$

Defining $\prod_{n=1}^{\infty}(\bar{q})^{n}$ by zeta function regularization $\left(\sum_{1}^{\infty} n=-1 / 12\right)$ yields

$$
I_{g}=\int_{M} \prod_{j} \frac{i x_{j} / 2}{\sin \left(i x_{j} / 2\right)} \frac{(\bar{q})^{-1 / 12}}{\prod_{n=1}^{\infty}\left(1-(\bar{q})^{n} e^{x_{j}}\right) \prod_{n=1}^{\infty}\left(1-(\bar{q})^{n} e^{-x_{j}}\right)} .
$$

If $p_{1}(M)=0$ then the identity between (21) and (17) is guaranteed by the following representation of the $\sigma$-function:

$$
\sigma(z ; \bar{\tau})=e^{\eta_{1} z^{2}} \frac{\sin (\pi z)}{\pi} \prod_{1}^{\infty}\left(1-(\bar{q})^{n} e^{2 \pi i z}\right) \prod_{1}^{\infty}\left(1-(\bar{q})^{n} e^{-2 \pi i z}\right) \prod_{1}^{\infty}\left(1-(\bar{q})^{n}\right)^{-2},
$$

where $\eta_{1}$ is defined in [23, p. 446].

To construct the Euler and the Hirzebruch indices we use the isomorphism between the exterior algebra and the tensor product of the spinor algebra with itself. This correspondence is well understood in finite dimension and there seems to be a formal infinite dimensional analogue. Let $\phi$ be a spinor and let $\varrho$ be a dual spinor. The tensor product $\phi \varrho$ is a bispinor which may be decomposed into a complete series of gamma matrices and their antisymmetric products:

$$
\phi \varrho \propto \operatorname{tr}(\varrho \phi)+\sum \gamma^{\mu} \operatorname{tr}\left(\varrho \gamma^{\mu} \phi\right)+\sum_{\mu<v} \gamma^{\mu} \gamma^{v} \operatorname{tr}\left(\varrho \gamma^{\mu} \gamma^{v} \phi\right)+\ldots
$$


This is the standard correspondence between spinors and the exterior algebra via the association of the wedge products $d x^{1} \wedge d x^{2} \wedge \ldots \wedge d x^{k}$ with the antisymmetric products of gamma matrices.

This observation is used in the following way. The canonical anticommutation relations for the fermions in the $N=1 / 2$ right moving sector are $\left\{\psi^{\mu}(\sigma), \psi^{v}\left(\sigma^{\prime}\right)\right\} \propto g^{\mu \nu} \delta\left(\sigma-\sigma^{\prime}\right)$. This means that the $\psi^{\prime}$ s are the gamma matrices in loop space. The carrier of the representation space for the gamma matrices are the spinors on loop space. We now introduce a right moving fermionic Ramond superfield which transforms as a covector

$$
N_{\mu}=\eta_{\mu}+\theta \varphi_{\mu} .
$$

The canonical commutation relations will tell us that the $\eta$ 's are dual to the "gamma" matrices, and therefore the carrier space for the representations are the dual spinors. The state created by the combination of $\psi$ 's and $\eta$ 's will be spinors tensored with the dual spinors. These are just the exterior algebra. One can make this identification more explicit by writing down the tangent space in terms of oscillators and comparing it with the Hilbert space generated by looking at the oscillator content of the fermions $\psi$ and $\eta$. The fermion parity in the right moving sector $(-1)^{F_{\mathrm{R}}}$ may be interpreted as multiplication of the bispinor by $\gamma_{5}$ on the left. Likewise, the fermion parity in the left moving sector $(-1)^{F_{\mathrm{L}}}$ may be interpreted as multiplication of the bispinor by $\gamma_{5}$ on the right.

The Euler characteristic compares forms of even degree with odd degree, therefore, it may be computed heuristically as

$$
\chi=\operatorname{Tr}(-1)^{F_{\mathrm{L}}+F_{\mathrm{R}}} .
$$

In the supersymmetry approach one calculates the above by having both the left and right moving sectors satisfying periodic boundary condition in the temporal direction.

In finite dimension the Hirzebruch signature compares self dual forms with anti-self dual forms. In Dirac language, the duality transformation is implemented by multiplication by $\gamma_{5}$ on the left. The associated index is formally

$$
\tau=\operatorname{Tr}(-1)^{F_{\mathrm{R}}} .
$$

What we are then led to calculate is the path integral with periodic temporal boundary conditions for both $\psi$ and $\eta$ in the case of the Euler number, and with periodic $\psi$ and anti-periodic $\eta$ in the case of the Hirzebruch signature [7, 8]. In analogy with (7) these path integrals compute respectively:

$$
\begin{aligned}
& I_{\chi}=\operatorname{Tr}(-1)^{F_{L}+F_{\mathrm{R}}} q^{L_{0}+\varepsilon}(\bar{q})^{\bar{L}_{0}+\bar{\varepsilon}}, \\
& I_{\tau}=\operatorname{Tr}(-1)^{F_{\mathrm{R}}} q^{L_{0}+\varepsilon}(\bar{q})^{\bar{L}_{0}+\bar{\varepsilon}},
\end{aligned}
$$

where now $L_{0}+\varepsilon$ and $\bar{L}_{0}+\bar{\varepsilon}$ contain contributions from the full system of two dimensional fields $X, \psi$, and $\eta$. As in the supersymmetric quantum mechanical case, the particular boundary conditions chosen to calculate the Euler number give rise to a left handed $N=1 / 2$ supersymmetry [8]. By expressing $\bar{L}_{0}+\bar{\varepsilon}$ as the square of this new supersymmetry, we can infer as before that only states with $\bar{L}_{0}+\bar{\varepsilon}=0$ contribute to the trace. So altogether only the zero modes should enter 
the expression for $I_{\chi}$. By explicitly carrying out the functional integration we obtain:

$$
\begin{aligned}
I_{\chi}= & \frac{1}{(2 \pi)^{d / 2}} \int d^{d} X_{0} d^{d} \psi_{0}\left[\operatorname{det}^{\prime}(-\partial \bar{\partial}+\mathscr{R} \bar{\partial})\right]^{-1 / 2} \\
& \times\left[\operatorname{det}^{\prime}(\bar{\partial})\right]^{1 / 2}[\operatorname{det}(-\partial-\mathscr{R})]^{1 / 2} .
\end{aligned}
$$

This expression directly simplifies into

$$
I_{\chi}=\frac{1}{(2 \pi)^{d / 2}} \int d^{d} X_{0} d^{d} \psi_{0}[\operatorname{det}(-\mathscr{R})]^{1 / 2},
$$

which as we promised only contains the contribution from the zero modes. Integrating over the fermionic zero modes $\psi_{0}$ leaves us with the Euler characteristic of $\mathscr{L}(M)$, which coincides with the Euler number of $M$ itself, according to the Lefshetz fixed-point theorem:

$$
I_{\chi}=\chi(M)=\int_{M} \prod_{j}\left(-x_{j}\right) .
$$

We remark that in this case we do not need to impose $p_{1}(M)=0$ in order to have a well defined index: the Euler number of a manifold is defined even if the manifold does not admit a spin structure (or a string structure in the case of the loop space). Possible inconsistencies due to the use of spinors to obtain the result should cancel out in the final expression, as they do.

Performing the path integral for the Hirzebruch signature gives rise to the same formula as in (29), but the determinant arising from the left handed fermions is to be evaluated over states with anti-periodic boundary conditions in $\tau$ :

$$
[\operatorname{det}(\partial+\mathscr{R})]^{1 / 2}=\prod_{j} \prod_{\Lambda}\left(n \bar{\tau}+m-\frac{1}{2}-2 i \tau_{2} x_{j}\right) .
$$

By using properties of elliptic functions [23] and defining $z_{j}=2 i \tau_{2} x_{j}$, (32) can be seen to be equal to

$$
\begin{aligned}
& {\left[\sqrt{2} \eta_{+}(\bar{q})\right]^{d} \prod_{j} \exp \left(\frac{1}{2} e_{1} z_{j}\right) \sigma_{1}\left(z_{j}\right)} \\
& \quad \times \exp \left\{-\sum_{j} \sum_{\Lambda}\left[\frac{z_{j}}{n \bar{\tau}+m-1 / 2}+\frac{1}{2}\left(\frac{z_{j}}{n \bar{\tau}+m-1 / 2}\right)^{2}\right]\right\},
\end{aligned}
$$

$\sigma_{1}$ being the elliptic generalization of the cosine function, defined in terms of the $\sigma$ function as $\sigma_{1}(z)=\exp \left(-\eta_{1} z\right) \cdot \sigma\left(z+\frac{1}{2}\right) / \sigma\left(\frac{1}{2}\right)$. The quantity $e_{1}=\wp(1 / 2)$ is discussed in $[23$, p. 443]. Combining the various pieces together we then obtain the following expression for the Hirzebruch signature of $\mathscr{L}(M)$ :

$$
I_{\tau}=\int_{M}\left(\prod_{j} 2 \cdot \frac{i x_{j}}{2 \pi} \cdot \frac{\sigma_{1}\left(i x_{j} / 2 \pi ; \bar{\tau}\right)}{\sigma\left(i x_{j} / 2 \pi ; \bar{\tau}\right)}\right) \cdot\left(\frac{\eta_{+}(\bar{q})}{\eta_{-}(\bar{q})}\right)^{d} .
$$

It is natural to interpret the ratio of the two sigma functions as the elliptic generalization of the circular cotangent. By using the representation (22) for the sigma function, we may rewrite the result in the following form:

$$
I_{\tau}=\int_{M} \prod_{j}\left(\frac{i x_{j}}{\tan \left(i x_{j} / 2\right)}\right) \cdot \prod_{1}^{\infty} \frac{\left(1+(\bar{q})^{n} e^{-x_{j}}\right)\left(1+(\bar{q})^{n} e^{x_{j}}\right)}{\left(1-(\bar{q})^{n} e^{-x_{j}}\right)\left(1-(\bar{q})^{n} e^{x_{j}}\right)} .
$$


It is straightforward to verify that this result agrees with what one would obtain by naively using the Atiyah-Bott theorem for the character valued Hirzebruch signature:

$$
\tau_{g}=\int_{N_{b}} \prod_{j} \frac{x_{j}}{\tan \left(i x_{j} / 2\right)} \cdot \prod_{r} \cot \left(\bar{\theta}_{r} / 2\right),
$$

where the same notation as in (19) has been used. Once again the manifold $M$ is not required to satisfy $p_{1}(M)=0$ in order for this index to be defined. Nevertheless in the course of the computations it is necessary to carry out a few regularizations. Even though these regularizations are compatible with the analyticity and the modular properties that we want to preserve, still a vanishing first Pontrjagin class and first Chern class would make all of the expressions that appear at intermediate steps of the computations completely finite, making possible a "careless" manipulation of the infinite sums and products.

In conclusion, we have shown how to use two-dimensional $N=1 / 2$ supersymmetric theories to probe the topology of the loop space of compact Riemannian manifolds. The existence of a group action on $\mathscr{L}(M)$ makes it possible to split the infinite dimensional kernels that appear in the definitions of various indices into finite dimensional representations of the group action. The results that we obtain suggest that the finite dimensional character-valued index theorems hold true when naively applied to the infinite dimensional manifold $\mathscr{L}(M)$, provided certain topological restrictions are satisfied by $M$ in the case of certain indices.

In principle this technique may have more general applications. It is possible, for example, to study a bosonic string with the conformal ghosts. The presence of these fermionic fields allows the construction of various infinite complexes, that may be studied by defining the proper cohomologies. It may be possible, for example, to interpret the partition function for the bosonic string in the critical dimension as the index of the BRST operator, thus providing an alternative proof of the no-ghost theorem. This approach is probably connected with the proof of the no-ghost theorem given by Frenkel et al. [29] who on the wave of the work by Banks and Peskin [30] constructed the semi-infinite cohomology generated by the ghost modes. Singer [31] points out that the proof of the no ghost theorem given by Frenkel et al. can be interpreted as the fixed point formula for the $S^{1}$-index theorem for $\bar{\partial}$ and the signature operator on the space Diff $\left(S^{1}\right) / S^{1}$, a Kähler manifold as defined in $[32,33]$.

M. Goodman has also been thinking about some of these issues.

Acknowledgements. O.A. would like to acknowledge general discussions with Iz Singer about index theorems and elliptic genera. P.W. would like to thank the people at IHES and particularly A. Connes for discussions and comments. M.M. would like to thank the UCB-LBL theory group for making possible a productive visit. O.A. would like to thank the A.P. Sloan Foundation for their generous support.

\section{References}

1. Atiyah, M., Bott, R.: The Lefschetz fixed-point theorem for elliptic complexes. I. Ann. Math. 86, 374 (1967)

2. Atiyah, M., Bott, R.: The Lefschetz fixed-point theorem for elliptic complexes. II. Ann. Math. 88, $451(1968)$

3. Atiyah, M., Segal, G.B.: Index of elliptic operators. II. Ann. Math. 86, 531 (1968)

4. Atiyah, M., Singer, I.: Index of elliptic operators. III. Ann. Math. 87, 546 (1968) 
5. Witten, E.: Global anomalies in string theory. In: Argonne symposium on geometry, anomalies, and topology. Bardeen, W.A., White, A. (eds.). New York: World Scientific 1985

6. Alvarez-Gaumé, L.: Supersymmetry and the Atiyah-Singer index theorem. Commun. Math. Phys. 90, 161 (1983)

7. Friedan, D., Windey, P.: Supersymmetric derivation of the Atiyah-Singer index theorem and the chiral anomaly. Nucl. Phys. B 235, 395 (1984)

8. Goodman, M.: Proof of the character-valued index theorems. Commun. Math. Phys. 107, 391 (1986)

9. Alvarez, O., Killingback, T., Mangano, M., Windey, P.: The index of the Ramond operator. Unpublished, Santa Barbara, August 1985

10. Witten, E.: Elliptic genera and quantum field theory. Commun. Math. Phys. 109, 525 (1987)

11. Schellekens, A., Warner, N.: Anomalies and modular invariance in string theory. Phys. Lett. 177 B, 317 (1986)

12. Schellekens, A., Warner, N.: Anomaly cancellation and self-dual lattices. Phys. Lett. 181 B, 339 (1986)

13. Schellekens, A., Warner, N.: Anomalies, characters, and strings. Nucl. Phys. B (submitted)

14. Pilch, K., Schellekens, A., Warner, N.: Path integral calculation of string anomalies. MIT preprint, 1986

15. Landweber, P.S., Stong, R.: Circle actions on spin manifolds and characteristic numbers. Commun. Math. Phys. (to appear)

16. Ochanine, S.: Sur les genres multiplicatifs définis par des intégrales elliptiques. Topology (to appear)

17. Zagier, D.: A note on the Landweber-Stong elliptic genus. in P. S. Landweber, editor Elliptic Curves and Modular Forms in Algebraic Topology, IAS, to appear

18. Killingback, T.P.: World-sheet anomalies and loop geometry. Nucl. Phys. (to appear)

19. Freed, D.: Determinants, torsion, and strings. Commun. Math. Phys. 107, 483 (1986)

20. Cecotti, S., Girardello, L.: Functional measure, topology, and dynamical supersymmetry breaking. Phys. Lett. 110 B, 39 (1982)

21. Cardy, J.: Operator content of two dimensional conformally invariant theories. Nucl. Phys. B 270 [FS16], 186 (1986)

22. Alvarez, O., Killingback, T., Mangano, M., Windey, P.: The Dirac-Ramond operator in string theory and loop space index theorems. Proc. Conf. on Nonperturbative Methods in field Theory Jan 87, Irvine, Univ. of California, Ed: H. Hamber, Proceedings Supplements Section Nucl. Phys. B

23. Whittaker, E.T., Watson, G.N.: A course of modern analysis. Cambridge: Cambridge University Press 1980

24. Candelas, P., Horowitz, G., Strominger, A., Witten, E.: Vacuum configurations for superstrings. Nucl. Phys. B 258, 46 (1985)

25. Gross, D.J., Harvey, J.A., Martinec, E., Rohm, R.: The heterotic string. I. Nucl. Phys. B 256, $253(1985)$

26. Gross, D.J., Harvey, J.A., Martinec, E., Rohm, R.: The heterotic string. II. Nucl. Phys. B 267, 75 (1986)

27. Green, M.B., Schwarz, J.H.: Anomaly cancellation in supersymmetric $D=10$ gauge theory require $S O(32)$. Phys. Lett. $149 \mathrm{~B}, 117$ (1984)

28. Friedan, D.: Non-linear $\sigma$-models in $2+\varepsilon$ dimensions. Phys. Rev. Lett. 45, 1057 (1980)

29. Frenkel, I., Garland, H., Zuckerman, G.: Semi-infinite cohomology and string theory. Proc. Nat. Acad. Sci. (USA) 83, 8442 (1986)

30. Banks, T., Peskin, M.E.: Gauge invariance of string fields. Nucl. Phys. B 264, 513 (1986)

31. Singer, I.M.: Private communication

32. Freed, D.: Ph.D. thesis, University of California, Berkeley (1985)

33. Bowick, M.J., Rajeev, S.G.: String theory as the Kähler geometry of loop space. Phys. Rev. Lett. 58, 535 (1987)

Communicated by A. Jaffe

Received March 13, 1987 